\title{
Trajectoires, filières, mythes. Les parcours migratoires des Italiens du canton de Vaud (Suisse)
}

\section{Marina Marengo, Arezzo}

\section{Introduction}

Derrière les motivations retenues par les filtres de l'officialité, les parcours migratoires cachent souvent une grande épaisseur de contenus. Cette richesse vient de l'expérience d'individus qui, par leurs parcours de vie, offrent au chercheur une matière complexe où l'ici et l'ailleurs cohabitent, se superposent, se bousculent. Les histoires de vie de ces immigrés sont bien sûr parsemées d'obstacles économiques, politiques, administratifs et sociaux souvent difficiles à gérer, ici comme «là-bas». Ces histoires, construites en faisant souvent recours à un imaginaire riche - parfois même trop abondant et presque toujours contradictoire -, constituent pour le chercheur une source d'informations précieuses car elles ne sont jamais banales. Dans ce travail nous nous proposons d'analyser, à l'appui d'histoires de vie d'Italiens résidant dans le canton de Vaud, la façon dont ces mêmes immigrés permettent à leur imaginaire de ressurgir, mais aussi de révéler les références aux mythes - de la Terre-Mère, du voyage, de la recherche de l'Eldorado, du retour - rattachés aux itinéraires migratoires qui nourrissent leur quotidien et leurs histoires.

Si des études scientifiques ou de vulgarisation ont mis en valeur certaines spécificités, surtout historiques, de la communauté italienne du canton de Vaud et de Suisse Romande, la parole n'a pas souvent été offerte à ces membres (Alleman-GHionda \& MeYer-Sabino 1992; Bolzmann, Fibbi \& Vial 1997; ECAP 1992; FibBI \& Piguet 1995; Meyer-Sabino 1987; Perrenoud 1990; Roche \& De Donno 2000). C'est en partie dans le dessein de leur donner la parole, pour se raconter et pour raconter leur histoire de l'immigration italienne de l'après-guerre que ce travail a été fait. Il ne s'agit pas de l'objectif originel de cette recherche, surtout centré sur l'individuation des caractéristiques géographiques de la communauté italienne et moins axé sur les pratiques et la parole des migrants en question. Ce n'est qu'au fil des rencontres, des discussions, de résultats scientifiques qui sentaient le déjà-vu, que le projet initial a évolué en allant vers les migrants, en cherchant à rendre à ces Italiens leur statut d'individus à part entière. Les entretiens et les histoires de vie utilisés dans le présent article ont été conduits et récoltés de 1993 à 1999 parmi les Italiens résidant dans le canton de Vaud (MARENGo 2001).

\section{Le parcours de recherche et quelques résultats}

Partis de schémas et de connaissances scientifiques «certaines» sur les phénomènes migratoires, nous avons dû nous obliger à revoir nos certitudes et à nous former tout en poursuivant notre chemin de recherche. Les connaissances et les compétences acquises "en route» nous ont bien confirmé, que, pour chercher à pénétrer dans l'écheveau des parcours migratoires, il nous fallait abandonner l'idée de pouvoir les mesurer. Si quelques velléités quantitatives nous animaient encore, les acteurs de terrain nous ont bien aidés à les dépasser, à ne pas nous conformer au «Diktat» de vouloir à tout prix mesurer un phénomène aussi opaque que les trajectoires migratoires. Par les reconstructions et les constructions en acte de ces mêmes trajectoires, nos témoins nous ont non seulement informée mais aussi formée à la découverte et à la reconstruction «bribe par bribe» de cette matière dense que sont les parcours migratoires des Italiens du canton de Vaud, ainsi qu'aidée à définir une grille de lecture de cet objet d'étude opaque et sinueux.

Au fur et à mesure que cette grille se construisait, il nous paraissait de plus en plus clair que les Italiens mobilisés dans la recherche étaient en train de nous informer sur l'existence d'un mode de vie centré sur plusieurs références spatiales, sociales et culturelles, qui ne pouvaient en aucune manière s'apparenter aux modèles de sédentarité et de mobilité "reconnus» par les académiciens et les gens communs. Ce mode de vie centré sur des ancrages réels ou cristallisés dans la mémoire et régulièrement «re-vivifiés», est axé sur une mobilité et un mouvement qui prennent leur source dans des racines «ancestrales» ou actuelles. Dans ce nouveau schéma de vie, que devient l'espace? Il devient un réseau fondé sur des références spatiales concrètes et quotidiennes et sur des références symboliques qui, malgré les obligations du quotidien et de la vie réelle, ne s'effacent jamais. L'ensemble de ces références sont constitutives de l'espace du migrant et de son mode de vie entre sédentarité et mobilité anciennes et nouvelles. Il s'agit d'un mode de vie fait

Le présent article constitue la suite et l'approfondissement d'une publication parue dans les actes du Colloque CLUSE de Neuchâtel en 1998 (MARENGo 2000). 
de ruptures, ces mêmes ruptures qui sont parfois incompréhensibles aux non-migrants, à tous ceux qui ne comprennent pas la «non conformation» du migrant à un modèle sédentaire. Ce schéma, apparemment révolutionnaire, n'est qu'un tout premier exemple, le plus ancien peut-être, des nouveaux modes de vie qui commencent à s'affirmer et à s'ancrer dans nos sociétés actuelles. Le migrant a été et continue d'être une sorte d'«éclaireur» pour ces mêmes sociétés qui, pourtant, n'arrêtent pas de le considérer «autre» et «hors norme» par rapport au modèle de sédentarité «monolithique» dominant.

Le migrant, l'«homme porteur d'un ailleurs» plus ou moins lointain, nous enseigne que de nos jours être pluriel risque bien être un atout, que la (re)construction de nos identités nous ouvre au principe de la multiplicité et de l'altérité:

«L'espace particulier que définit la migration semble doté de curieuses propriétés élastiques, puisqu'il opère des rapprochements autrement considérés comme insolites, qu'il bouleverse l'ordre visible du monde» (VIDELIER 1993: 183);

mais en même temps cet espace possède une rigidité ce qui n'est pas une contradiction malgré les apparences - fondatrice, une rigidité qui s'est construite sur la sédimentation d'expériences et de connaissances «du monde» acquises tout au long des trajectoires migratoires.

\section{L'ancrage fondamental: la Terre-Mère}

Le discours sur la décision migratoire, les motivations constitutives de ce choix, les stratégies identitaires de l'être sédentaire devenu mobile sont utilisés dans ce contexte pour comprendre l'enracinement «en tant que rapport affectif et identificatoire de l'homme à la terre» (Genestier 1989: 50). La décision migratoire, tout comme les questions d'enracinement, paraissent être prédominantes et fondatrices par rapport à l'usage habituel qui en est fait (politique, administratif ou scientifique).

Qu'ils soient de première ou de troisième génération, qu'ils soient nés en Suisse ou qu'ils soient arrivés dans ce pays déjà adultes, dans leurs discours les Italiens mobilisent tous les liens affectifs et mémoriels qui sont constitutifs, voire fondateurs, de leur identité. L'ici et l'ailleurs - mais où est l'ici et où est, par contre, l'ailleurs? (MARENGo \& AlAimo 2000) - cohabitent, se superposent, se bousculent en nous permettant d'apercevoir la complexité d'une identité souvent difficile à assumer mais qu'ils ont pourtant contribué à forger eux-mêmes, en déployant des stratégies identitaires jaillies de décisions migratoires qui leur appartiennent, tout comme leurs projets de vie.
D’un côté les références spatiales mobilisées sont liées à une conception de l'enracinement centré sur l'ancestralité, héritage des sociétés rurales d'origine ou dont les principes fondateurs ont été à la base de leur éducation:

"Quand je suis descendu en octobre [...] j'ai trouvé les figues de Barbarie. LES FIGUES DE BARBARIE!! Quand j'étais là quand j'étais jeune il y avait dans la campagne les figues de Barbarie et le matin elles étaient fraîches. Quand j'ai construit la maison j'ai fait planter ces figues de Barbarie [...] Je m'y suis mis avec le couteau et avec le pain, là [...] la joie que j'ai éprouvé à sentir cette saveur-là et le plaisir de les prendre moi-même de me les couper. C'était tout comme je le faisais il y a trente-deux ans» (Salvatore, 51 ans, 1 ère génération).

De l'autre côté, et avec la prédominance de la dimension temporelle sur la dimension spatiale - la «temporalisation de l'espace» dont parlait MoscovicI (1983: 262) - l'enracinement évoqué par les interviewés possède une valeur et une épaisseur aussi spirituelle que concrète. Cette conception de l'enracinement est proche des valeurs existentielles et pratiques: elle est historique et «moderne» (ELIADE 1969: 174):

«D'un côté il y a vraiment la nécessité de retourner aux origines, c'est-à-dire que tu as ce sens de l'appartenance. Moi, par exemple, je l'ai avec la mer, avec la mer et des fois aussi avec la nature, même si la nature estivale en Sicile paraît plus agressive que familière. J'ai la nécessité de cette chaleur horrible, de ce soleil aveuglant, de cette mer chaude. Ce sont des nécessités [...]» (MATILDE, 39 ans, 1ère génération).

La mobilisation synchronique des deux conceptions de l'enracinement peut être expliquée par la duplicité des dimensions spatio-temporelles qui cohabitent et parfois se bousculent tout au long du parcours migratoire ainsi que dans le projet de vie de nos témoins. La circularité de l'espace et la sérialité du temps de la tradition - fondées sur une conception spatialisante dominante et "encadrant» ainsi le temps, ancrée à la Terre-Mère et à la cyclicité du temps, ressurgissent aussi souvent que le projet de ces migrants le demande. Elles ressurgissent, mais sont couplées à une conception de spatialité dilatée et où la non-contiguité spatiale est dominante - illimitée et non limitant l'action humaine -, ainsi qu'à une temporalité devenue linéaire - le temps devenu fléché et épris de vitesse - qui constituent la conception «moderne» de l'espace-temps (LÉvY-Leblond 1983). Cette cohabitation contradictoire est autant mise en évidence dans les discours concernant directement l'identité que dans les rapports des Italiens à la sédentarité/mobilité et à l'enracinement/ déracinement. 


\section{Le mythe du voyage: à la recherche de l'Eldorado}

En s'appuyant sur le postulat qu'une migration n'est pas seulement économique, dans le sens que la question économique et matérielle n'est que l'une des nombreuses motivations qui portent l'individu à un choix de mobilité - même si dans un cadre d'officialité elle reste toujours dominante - il est possible de tenter d'analyser les discours de nos témoins sous un angle différent, bien plus individuel - voire intime - par rapport aux discours couramment tenus sur les migrations internationales par les autorités comme par les scientifiques ainsi que, dans certains cas, par les immigrés eux-mêmes (Braun 1970; SAYAD 1975, 1979).

Si les extraits d'entretiens présentés jusqu'ici nous ont permis d'analyser l'attachement ou mieux l'enracinement en mobilisant le concept de fondation et les mythes rattachés à la Terre-Mère, les motivations à la migration ainsi que la décision d'entrer dans un projet de mobilité - donc le passage à l'acte - nous permettent d'évoquer le mythe du voyage. Les Italiens mobilisés ne sont ni des explorateurs, dans le sens courant du terme, ni des conquérants mais plutôt, dans certains cas, des «audaces» qui ont pris le risque de tout bousculer dans leur vie par un choix difficile et à contrecourant, où le goût de l'aventure est sans doute présent, qui témoigne aussi d'une envie, d'un besoin de recommencer qui ne pouvait pas se concrétiser selon leurs désirs dans l'espace d'origine:

«Je suis le seul à l'étranger dans ma famille [...] C'est moi qui ai choisi [...] J'ai fait les études jusqu'à la maturité technique. Je voulais m'inscrire à l'université, en architecture. Mais le manque de travail qui existait déjà à l'époque, je parle de '69, m’a porté à choisir de faire le service militaire, dans la Marine, 24 mois [...] j'ai été congédié avec le grade de sergent major. A l'époque un sergent major gagnait plus qu'un fonctionnaire d'Etat [...] Je gagnais beaucoup plus que mon père...qui était fonctionnaire d'Etat. Cela m'a aussi porté à tout abandonner [...] et en plus des divergences avec mon milieu [...] j'ai toujours été un type direct, franc, sincère [...] Tout cela m'a porté à tout laisser, à venir en Suisse et y recommencer une nouvelle vie» (TANO, 47 ans, 1ère génération).

Le projet migratoire peut aussi constituer, du moins au début, un non-projet, dans le sens que le migrant, ou mieux l'individu qui s'est auto-investi de l'habitus d'être de la mobilité, est conscient de son besoin de mouvement afin de réaliser ses aspirations et d'apaiser ses inquiétudes mais n'a pas défini au départ un projet migratoire. La casualité, l'opportunité qui se présente sans trop l'avoir cherchée, est souvent à l'origine de ce mouvement spécifique: la migration. Cela ne veut pas dire que la recherche de l'Eldorado ne soit pas présente mais plutôt qu'elle ne se manifeste et ne s'actualise pas toujours avant tout autre type de motivation:

«Je viens de Modena. Je suis venu en Suisse à 18 ans [...] pour rendre visite à ma soeur et j'y suis resté [...] Je n'avais pas décidé d’émigrer. Après j’ai cherché un travail, car il faut bien vivre. et puis j'ai connu les copains d'une association sportive de football [...] et je suis toujours ici» (GIAсомо, 66 ans, 1ère génération).

\section{Le nouvel ancrage: un rite de re-fondation?}

Sédentarité et mobilité dans les parcours de vie des Italiens ne s'excluent pas, elles cohabitent et sont souvent remises en question par ces êtres possédant des identités complexes. En tout cas, elles ne s'annulent pas. Et cela car l'enracinement «fondateur» est, la plupart du temps, suivi d'un nouvel ancrage, un rite de fondation que le migrant accomplit dans son nouvel espace de vie où il ne trouve pas «son Eldorado» tout fait. Il le construit en revanche au jour le jour, par ses actions et ses pratiques, en jetant au fur et à mesure les bases d'un nouvel enracinement, qui devient l'ancrage le plus concret, celui où il participe activement à la construction d'une société et dans lequel il peut se penser en devenir:

«A Terni où je travaillais j'avais un travail, un bon boulot, mais je savais que je n'aurais jamais pu faire une carrière parce que j'étais de gauche [...] Alors j'ai demandé quelques renseignements et je suis venu ici. J'ai recommencé comme manœuvre même si j’avais un métier [...] J'ai remonté toute la pente [...] Maintenant je suis indépendant, j'ai une entreprise de mécanique et $\mathrm{j}$ 'ai la responsabilité de plusieurs personnes» (GıovanNı, 65 ans, 1ère génération).

Cela ne veut pourtant pas dire que l'ancrage premier du migrant disparaît ou est abandonné: les deux cohabitent et peuvent se superposer en donnant origine à un espace de vie qui n'est plus que simple territoire (PIolle 1990). Il se fait réseau et dans ce réseau le migrant organise sa vie, formule son (ses) projet(s), donne de nouveaux sens à ses besoins et à ses angoisses de sédentarité et de mobilité (Chivallon 1996). Cependant la cohabitation de référents divers n'est pas seulement spatiale, elle est aussi temporelle: d'un côté il y a le passé fondateur, rattaché à l'espace du souvenir et de la fondation première, tandis que de l'autre côté se prépare le futur à construire, lié à l'espace du devenir, de toute nouvelle fondation possible, l'espace où il faut se mettre à courir (SAYAD 1975).

\section{La condition migrante: entre stabilité et dynamisme}

La dualité spatio-temporelle constitue bien la base de l'identité de nos témoins: «elle constitue certainement 
une assise du rapport au monde et de la représentation de soi-même» (Genestier 1989: 51). Etre immigré ou être migrant ne signifie pas construire son existence sans aucun point de repère, dans l'errance dirait MAFFESOli (1997), sans lieux d'ancrage. Claire et nette ou bien encore opaque et incertaine, c'est une nouvelle manière d'aborder la stabilité et le dynamisme qui se définit dans les discours de nos témoins. La première est fondatrice, mais pas immobile malgré les références à la tradition et à la Terre-Mère: elle se fait dynamique, car à chaque visite, à chaque retour elle se présente sous un nouvel angle parfois négatif ou critique, se revitalise, se dilate et se complexifie tout en gardant toujours sa fonction d'ancrage et de point de repère:

«Quand tu y habites pour plus de deux semaines ressortent toutes les choses négatives ... Je la vois aussi à travers le regard d'une personne qui habite en Suisse depuis 16 ans et donc par le regard d'une personne qui dit «mais il pourraient le faire mieux» [...] pour une personne qui n'y est pas née ... c'est une agression forte. Encore une fois la lumière, la chaleur, les odeurs, les bruits sont tous des agressions fortes et qui peuvent mettre mal à l'aise. Ce sont par contre les choses qui me mettent à l'aise» (MATILDE, 39 ans, 1ère génération).

La stabilité se situe même à la base de tout mouvement. Sans un ancrage, que l'on pourrait définir comme ancestral, les opportunités de mobilité et de formulation d'un projet de vie ne sont pas réalisables, voire envisageables. La mobilité, elle aussi, n'est pas synonyme de dynamisme car le seul mouvement dans l'espace ne donne pas à ces Italiens la possibilité de construire un projet de vie plein. La mobilité ne peut exister pour eux que s'il y a un point de départ toujours clair dans leur trajectoire, même s'il n'est que symbolique. Toute nouvelle fondation ne peut prendre sa source que dans un espace de fondation première ainsi que dans une histoire qui n'est pas que devenir et qui n'exclut pas l'immuabilité.

Cette hypothèse paraît être confirmée par les discours tenus par les deuxième et troisième générations. Ces jeunes sont des individus de la mobilité «malgré eux», car nés dans un projet de vie qui n'était pas le leur mais dont ils font partie, dans lequel ils sont insérés, de la même manière que les premières générations se sont retrouvées dans un projet de vie ancré dans la sédentarité qui n'était pas le leur mais celui de leurs parents ou de leurs ancêtres. Ces êtres de la mobilité "malgré eux» cherchent des repères, des ancrages qui correspondent à la recherche de la Terre-Mère ainsi que de la Terre Promise, cet espace dont ils ont hérité le mythe mais sur lequel ils n'ont accompli aucun acte fondateur et dont il n'ont jamais fait le deuil:
«Je voudrais aller en Italie parce que là-bas j'ai la terre. Ici je suis chauffeur ... tu vois, ce serait bien différent [...] pouvoir labourer la terre, ma terre. Ici je n'ai pas vraiment de repères malgré ma femme et mon fils» (PAOLO, 32 ans, 2ème génération).

\section{Le mythe du retour: réalité ou imaginaire?}

Une fois l'Eldorado atteint, une fois insérés dans l'espace et la société d'accueil, souvent non sans difficultés et souffrances, ces Italiens ont vu jaillir, subitement ou dans le temps, toutes les prémices d'un nouveau mythe: celui du retour à la Terre-Mère qui, d'espace clos, limité et limitant, s'est transformé petit à petit en espace de rassurement, de refuge, d'épanouissement dans la certitude d'un ancrage ancestral (ELIADE 1969; Boudoudou 1985).

Dans ce projet de vie ancrée entre la stabilité et la mobilité, le mythe du retour assume des connotations qui peuvent parfois être assez différentes sinon contradictoires (ACHESON 1993). Le «retour» définitif étant souvent irréalisable, c'est le retour temporaire, les vacances ou en tout cas les séjours de courte durée qui jouent le rôle de ressourcement identitaire et qui nourrissent le mythe. Nos témoins sont bien conscients qu'un retour définitif est souvent improbable, y compris une fois l'âge de la retraite atteint:

«[...] mais je ne peux pas penser à un vrai retour parce que ma femme parce que mes enfants et mes petits enfants sont ici. Bien sûr que je voudrais bien retourner à Modena mais c'est trop difficile» (Gıасомо, 64 ans, lère génération).

Un retour vraiment définitif à la Terre-Mère ne pourrait devenir une réalité qu'à la fin de leur parcours de vie:

«Mais comme j'ai toujours dit à ma fille [...] Si je devais partir, si je devais mourir pour le dire plus clair, tu ne devras faire autre chose [...] que disperser mes cendres dans le petit port [de Cefalù]» (TANo, 47 ans, lère génération).

Mais le mythe du retour multiplie dans certains cas sa puissance. Ce mythe est exploité «comme solution de rechange à la difficile insertion dans la société de la nation dans laquelle on est né» (CATANI 1983: 119) ou, en tout cas, où l'on a été éduqué:

«Je ne suis pas à l'aise ici. Mon père nous a éduqué à l'idée du retour. Déjà une fois l'école terminée j'ai fait plusieurs demandes pour travailler et vivre en Italie. Ça n'a pas marché mais si je dois trouver une solution parce que ici je ne suis pas bien. Les copains ici sont tous des Italiens, 
je parle surtout l'italien ... les Suisses c'est autre chose et la Suisse aussi même si j'y suis né. Je veux retourner sur ma terre» (PAOLO, 32 ans, 2ème génération).

Cette migration «à rebours», comme la définit CATANI (ibid) joue dans ce cas le rôle d'une bouée de sauvetage, d'une volonté de s'offrir un nouveau départ, un nouveau projet de vie, de définition de la part de ces êtres de la mobilité «malgré eux». Mais ces immigrés n'utilisent-ils pas ce projet de retour que comme miroir, que comme illusion qu'ils veulent à tout prix transformer en réalité?

\section{Conclusions et perspectives}

De ces quelques extraits tirés des histoires de vie des Italiens du canton de Vaud, des trajectoires d'approfondissement ultérieur des parcours migratoires se sont dégagées au fur et à mesure que l'analyse des extraits progressait. Traces incertaines encore peu creusées ou pistes déjà ouvertes, ces trajectoires de recherche pourraient permettre de mieux comprendre des projets migratoires fondés sur des choix souvent inusuels mais moins rares que ce que l'on pourrait croire.

L'importance des voies ouvertes par des chercheurs comme BraUn (1970) et SAYAD $(1975,1979)$, pour n'en citer que deux, est une fois de plus confirmée par nos témoins qui, grâce à leurs discours, nous ont permis de saisir une partie au moins des motivations migratoires possibles. L'indication la plus claire nous conduit en direction des aspirations individuelles qui «orientent» souvent les choix de mobilité des individus. Qu'il s'agisse d'une mobilité centrée sur un projet migratoire formulé d'avance ou bien d'un mouvement né à partir d'une occasion impromptue qui peut donner un nouveau tournant à une vie, les aspirations individuelles constituent une part fondamentale de la décision migratoire. Vouloir départager ces motivations des autres, surtout celles qui sont consignées dans les documents officiels, risque d'annuler ou de rendre moins crédible tout effort de compréhension. La saisie et l'analyse des «familles» de motivations les plus fréquentes, et qui se présentent avec plus de régularité et continuité, se révèlent donc nécessaires, malgré une complexité et une opacité tellement denses qu'il est parfois difficile d'aller un plus loin que les pistes déjà tracées.

Une autre piste ultérieurement dégagée dans ce travail concerne les «enfants de la migration». Encore enfants ou déjà adultes, ils ne peuvent que rarement se faufiler à travers les mailles problématiques d'un projet migratoire qu'ils n'ont pas élaboré. Le manque d'ancrages fondateurs, ou leur peu de force, suggère aux jeunes les plus pragmatiques d'apprendre à tirer le plus d'avantages possibles de leur situation en porte-à-faux. En revanche, ceux qui ressentent le plus ce manque d'enracinements «sûrs» se sentent diminués, au point de ne pas être à même de saisir les opportunités qui s'ouvrent à eux car renfermés dans un projet de vie d'autrui.

Enfin la dernière trajectoire est celle qui prend sa source dans cet imaginaire si opulent qu'il occupe parfois tant de place que les immigrés eux-mêmes ont du mal à l'assumer. Cependant la grande familiarité que ces individus ont avec l'aspect imaginaire des migrations et leurs capacités mythifiantes, peut suggérer des parcours de recherche qui leur attribuent enfin les premiers rôles d'acteurs de leur propre vie, en valorisant leur parole mais «en essayant toutefois de ne jamais s'instituer en porte-parole, sans jamais s'autoriser de la parole donnée» (BourdiEu 1991: 8-9).

\section{Bibliographie}

Acheson, J. (1993): Le retour au pays. - In: Collectif: Vers un ailleurs prometteur. - Genève: Institut universitaire d'études du développement (IUED) et Paris: Presses universitaires de France: 335-347.

Alleman-Ghionda, C. \& G. Meyer-Sabino (1992): Donne italiane in Svizzera. - Locarno: Dadò Editore. Bolzman, C., Fibbi, R. \& M. Vial (1997): Espagnols et Italiens proches de la retraite: structure et fonctionnement du réseau familial. - In: BédAY-Hauser, P. \& C. Bolzman (éds): On est né quelque part mais on peut vivre ailleurs. Familles, migrations, cultures, travail social. - Genève: Institut d'études sociales (IES). Boudoudou, M. (1985): «Mythe» du retour et imaginaire social. - In: Peuples Méditerranéens 31-32: 227-241.

Bourdieu, P. (1991): Préface. - In: Sayad, A.: L'immigration ou les paradoxes de l'altérité. - Bruxelles: De Boeck-Wesmael: 7-9.

Braun, R. (1970): Sozio-kulturelle Probleme der Eingliederung italienischer Arbeitskräfte in der Schweiz. - Erlenbach-Zürich: Eugen Rentsch Verlag.

Catani, M. (1983): L'identité et les choix relatifs aux systèmes de valeurs. - In: Peuples Méditerranéens 24: 117-126.

Chivallon, C. (1996): Repenser le territoire, à propos de l'expérience antillaise. - In: Géographie et Cultures 20: $45-54$.

ECAP (1992): La mappa del disagio. Indagine sulle tipologie dei servizi sociali operanti in Svizzera a favore della comunità italiana e sulle manifestazioni di disagio. - Zürich: Fondazione ECAP.

Eliade, M. (1969): Le mythe de l'éternel retour. Paris: Gallimard.

Fibil, R. \& E. Piguet (1995): La contribution des immigrés au développement de leur pays d'origine, le cas des Italiens de Suisse. - Paris: OCDE. 
Genestier, P. (1989): Sous les pavés, les racines? - In: EspacesTemps 42: 50-57.

LÉVY-LEBLOND, J.-M. (1983): La structure de l'espacetemps (les relativités). - In: Collectif: L'espace et le temps aujourd'hui. - Paris: Seuil: 76-89.

MafFesoli, M. (1997): Du nomadisme. Vagabondages initiatiques. - Paris: Librairie Générale Française.

Marengo, M. \& A. Alaimo (2000): Gli itinerari della memoria. Il paesaggio della Sicilia nei racconti degli immigrati siciliani a Losanna ( $\mathrm{CH})$. - In: Cusimano, G. (éd.): La costruzione del paesaggio siciliano: geografi e scrittori a confronto. - Palermo: Annali della Facoltà di Lettere e Filosofia 12: 483-506.

MARENGO, M. (2000): Les trajectoires migratoires entre mythes et nouvelles identités. L'exemple des Italiens du canton de Vaud. - In: Centlivres, P. \& I. Girod (dir.): Les défis migratoires. Actes du colloque CLUSE «Les défis migratoires à l'aube du troisième millénaire», Neuchâtel, 1998. - Zurich: Editions Seismo: 441-449.

MARENGo, M.(2001): Les trajectoires migratoires: entre flux, filières et mythes. Le cas des Italiens du canton de Vaud. - Travaux et recherches de l'Institut 21, Lausanne: Institut de géographie

Meyer-SABino, G. (éd.) (1987): La generazione della sfida quotidiana. Studio sulla condizione dei giovani italiani in Svizzera. - Roma: Ente nazionale acli istruzione professionale (enaip).

Moscovici, S. (1983): L'espace, le temps et le social. In: Collectif: L'espace et le temps aujourd'hui. - Paris: Seuil: 261-272.

Perrenoud, M. (1990): Attitudes suisses vis-à-vis de l'immigration italienne après 1945. - In: Revue syndicale suisse $82: 4$.

Piolle, X. (1990): Mobilité, identités, territoires. - In: Revue de Géographie de Lyon 65, 3: 149-154.

Roche, S. \& M.-R. De Donno (2000): L'Italienne. Orbe: Campiche Editeur.

SAYAD, A. (1975): El Ghorba: Le mécanisme de reproduction de l'émigration. - In: Actes de la recherche en sciences sociales 2: 50-66.

SAYAD, A. (1979): Qu'est-ce que c'est un immigré. - In: Peuples Méditerranéens 7: 15-26.

VIDELIER, P. (1993): La migration comme configuration du monde. - In: Ethnologie française 23: 177-184.

\section{Résumé: Trajectoires, filières, mythes. Les parcours migratoires des Italiens du canton de Vaud (Suisse)} Les filtres de l'officialité de la migration, dans le pays d'origine comme dans celui d'accueil, ne permettent pas de saisir et de comprendre la grande épaisseur de contenus cachés dans les parcours migratoires. Dans la présente contribution nous nous proposons d'analyser, à l'aide d'histoires de vie d'immigrés italiens résidant dans le canton de Vaud, la façon dont ces immi- grés permettent à leur imaginaire de ressurgir ainsi que de révéler les références aux mythes de la «TerreMère», du voyage, de la recherche de la «Terre Promise», du retour, rattachés aux trajectoires migratoires qui nourrissent leur quotidien et leurs histoires.

\begin{abstract}
Careers and myths. Experiences of Italian migrants in the Canton of Vaud (Switzerland)

The bureaucracy involved in migration within the countries of origin and destination makes it difficult to put together the stories of individual migrants. This article therefore draws on the experiences made by Italian immigrants in the Canton of Vaud in an attempt to touch on the emotional processes involved in migration, processes which are strongly affected by images and myths created and fed by thousands of migrants before, be these images of Mother Earth, of the journey, of the search for the Promised Land and of the return thereafter.
\end{abstract}

\section{Zusammenfassung: Karrieren und Mythen. Zum Werdegang der italienischen MigrantInnen des Kantons Waadt (Schweiz)}

Das Verwaltungsprozedere bei der Einwanderung, welches sowohl im Herkunfts- wie auch im Ankunftsland die Geschichte jedes Emigranten und jeder Emigrantin vereinheitlicht, macht es meist unmöglich, das individuelle Profil dieser Personen zu erfassen. In diesem Artikel geht es darum, anhand der Geschichte einzelner im Kanton Waadt lebender italienischer ImmigrantInnen die Vorgänge zu untersuchen, die im Vorstellungsbereich dieser Personen ablaufen: ihr Bild von der «Mutter Erde», von der Reise, von der Suche nach dem «gelobten Land» und von der Rückkehr, Bilder, welche die zahllosen Migrationen in ihrer Geschichte geprägt haben.

Prof. Dr. Marina Marengo, Dipartimento di Teoria e Documentazione delle Tradizioni Culturali, Facoltà di Lettere e Filosofia in Arezzo, Università degli Studi di Siena, Viale Cittadini 33, I-52100 Arezzo.

e-mail:marengo@unisi.it

\section{Manuskripteingang/received/manuscrit entré le 22.6.2005}

Annahme zum Druck/accepted for publication/accepté pour l'impression: 23.8.2005 